\title{
Phase I/II study of verteporfin photodynamic therapy in locally advanced pancreatic cancer
}

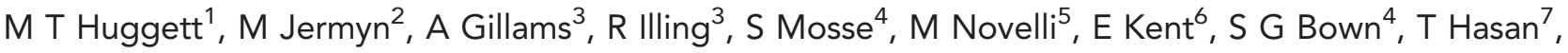
B W Pogue ${ }^{2}$ and S P Pereira*,1

${ }^{1}$ UCL Institute for Liver and Digestive Health, University College London, UCL Medical School-Royal Free Hospital Campus, U3 Floor, Pond Street, London NW3 2QG, UK; ${ }^{2}$ Thayer School of Engineering, Dartmouth College, Hanover, NH 03755, USA; ${ }^{3}$ Department of Radiology, University College Hospital, London, UK; ${ }^{4}$ National Medical Laser Centre, University College London, London, UK; ${ }^{5}$ Department of Pathology, University College Hospital, London, UK; ${ }^{6}$ UCLH Cancer Clinical Trials Unit, University College Hospital, London, UK and ' Wellman Center for Photomedicine, Massachusetts General Hospital, Boston, MA 02114, USA
\end{abstract}

Background: Patients with pancreatic cancer have a poor prognosis apart from the few suitable for surgery. Photodynamic therapy (PDT) produces localised tissue necrosis but previous studies using the photosensitiser meso-tetrahydroxyphenylchlorin (mTHPC) caused prolonged skin photosensitivity. This study assessed a shorter acting photosensitiser, verteporfin.

Methods: Fifteen inoperable patients with locally advanced cancers were sensitised with $0.4 \mathrm{mg} \mathrm{kg}^{-1}$ verteporfin. After $60-90 \mathrm{~min}$, laser light $(690 \mathrm{~nm}$ ) was delivered via single (13 patients) or multiple (2 patients) fibres positioned percutaneously under computed tomography (CT) guidance, the light dose escalating (initially $5 \mathrm{~J}$, doubling after each three patients) until $12 \mathrm{~mm}$ of necrosis was achieved consistently.

Results: In all, $12 \mathrm{~mm}$ lesions were seen consistently at $40 \mathrm{~J}$, but with considerable variation in necrosis volume (mean volume $3.5 \mathrm{~cm}^{3}$ at $40 \mathrm{~J}$ ). Minor, self-limiting extrapancreatic effects were seen in multifibre patients. No adverse interactions were seen in patients given chemotherapy or radiotherapy before or after PDT. After PDT, one patient underwent an RO Whipple's pancreaticoduodenectomy.

Conclusions: Verteporfin PDT-induced tumour necrosis in locally advanced pancreatic cancer is feasible and safe. It can be delivered with a much shorter drug light interval and with less photosensitivity than with older compounds.

\section{INTRODUCTION}

Pancreatic cancer. Adenocarcinoma of the pancreas is one of the top 10 leading causes of cancer deaths. In the United Kingdom, $\sim 8000$ people are diagnosed with the disease each year. Surgical resection is the only chance of cure and is only possible in a minority of subjects. Even after resection, the median survival is only $10-20$ months and only $12-35 \%$ of resected patients survive 5 years or more (Kinsella et al, 2008; Eeson et al, 2012; He et al, 2013; Turrini et al, 2013).

Palliative chemotherapy has been shown to improve both quality of life and survival in advanced pancreatic cancer, when compared with best supportive care. Combination regimens including FOLFIRINOX (oxaliplatin, irinotecan, fluorouracil, and leucovorin) have shown improved outcome compared with gemcitabine monotherapy (median overall survival 11.1 vs 6.8 months; hazard ratio $(\mathrm{HR})=0.57 ; P<0.001)$ (Conroy et al, 2011), while a phase III study of weekly intravenous albumin-bound paclitaxel (nab-paclitaxel) plus gemcitabine resulted in a significantly higher overall survival compared with gemcitabine monotherapy (8.5 vs 6.7 months; $\mathrm{HR}=0.72 ; P<0.001$ ), with survival rates of $35 \%$ vs $22 \%$ at 1 year (Von Hoff et al, 2013). The UK GEM-CAP trial reported a median survival in the combination capecitabine + gemcitabine group of 7.1 months compared with 6.2 months in those who received gemcitabine alone, with 1-year overall survival rates of $24.3 \%$ vs $22 \%(\mathrm{HR}=0.86 ; P=0.077)$

*Correspondence: Dr SP Pereira; E-mail: stephen.pereira@ucl.ac.uk

Received 29 October 2013; revised 5 January 2014; accepted 29 January 2014; published online 25 February 2014 (c) 2014 Cancer Research UK. All rights reserved 0007-0920/14 
(Cunningham et al, 2009). Trials of monoclonal antibodies and small molecule inhibitors have had limited success (Moore et al, 2007; von Wichert et al, 2008; Kindler et al, 2010). Overall, the long-term prognosis of the disease is poor with a 1-year survival rate of 15-39\% (Sant et al, 2009; Cronin-Fenton et al, 2011; Gong et al, 2011; Kuroda et al, 2013; Luo et al, 2013). For locally advanced disease, the median survival is $6-10$ months, although for those with metastatic disease at presentation, the median survival may be as low as 3-6 months.

Given these dismal results, a minimally invasive treatment capable of local destruction of tumour tissue with low morbidity may have a place in the treatment of unresectable disease.

Photodynamic therapy. Photodynamic therapy (PDT) is a way of producing localised tissue necrosis with light (most conveniently from a low-power laser), after prior administration of a photosensitising agent. It causes tissue necrosis by a non-thermal cytotoxic effect, thought to be mediated by singlet oxygen. As the biological effect is photochemical, not thermal, there is little damage to connective tissues such as collagen and elastin so helping to preserve the mechanical integrity of tissues, particularly hollow organs (Barr et al, 1987).

A pilot clinical trial of PDT in pancreatic cancer (the first in the world), using meso-tetrahydroxyphenylchlorin (mTHPC), was published by our group in 2002 (Bown et al, 2002). Sixteen patients with locally advanced cancers in the head of the pancreas were treated with PDT using light delivered directly into the tumours by laser fibres inserted through needles positioned percutaneously through the anterior abdominal wall under ultrasound and computed tomography (CT) guidance. All patients were found to have areas of tumour necrosis (up to a volume of $60 \mathrm{~cm}^{3}$ ) on CT within a few days of PDT. There was no treatmentrelated mortality and all but two patients were discharged from hospital within 10 days. It was concluded that PDT could produce necrosis in pancreatic cancers with an acceptable morbidity, although care was required for tumours invading the duodenal wall or involving the gastroduodenal artery. The median survival after PDT was 9.5 months (range 4-30, one patient alive at 31 months; median 12.5 months after treatment).

Verteporfin is a second-generation photosensitiser that has been licensed for $>10$ years for the treatment of age-related macular degeneration ( $\mathrm{Wu}$ and Murphy, 1999). There has been considerable interest in its potential for the management of ocular tumours, but relatively little investigation into its value for other oncological indications, aside from pre-clinical studies in oesophagus and vertebral breast metastases (Panjehpour et al, 2002; Lo et al, 2012).

Compared with the first-generation photosensitiser, mTHPC, the key attraction of verteporfin is its rapid clearance by excretion in bile. The peak tissue concentration of verteporfin is seen within an hour or two of administration, so light can be delivered with a much shorter drug light interval than the 2-4 days required with mTHPC (Lin et al, 1998; Fingar et al, 1999); and the period of skin photosensitivity is typically only $24 \mathrm{~h}$ (Houle and Strong, 2002a, b). In contrast, patients treated with mTHPC must avoid bright daylight for 7 days and avoid bright sunlight for 1 month. Pre-clinical studies of the pharmacokinetics and mechanisms of tissue killing with verteporfin have been developed extensively in a number of cancer models and show that while it has been largely considered a vascular damaging photosensitiser, it also has a potent cellular killing efficacy that is present when given sufficient time for transvascular permeation and diffusion in the tumour tissue (Chen et al, 2003, 2005a, 2005b; Samkoe et al, 2010; Glidden et al, 2012).

Another advantage of verteporfin is its strong absorption at $690 \mathrm{~nm}$, a wavelength at which light penetrates tissue better than at the shorter red wavelength absorption peaks of mTHPC and porfimer sodium. It has a weaker absorption peak at $630 \mathrm{~nm}$, which was previously used pre-clinically in the treatment of the canine oesophagus, but the deeper penetration and the stronger absorption at $690 \mathrm{~nm}$ are more appropriate for treating tumours in solid organs like the pancreas (Panjehpour et al, 2002). The safety of interstitial PDT using verteporfin was investigated by our group in the Syrian golden hamster (Ayaru et al, 2007). Light doses of 10 and $25 \mathrm{~J} \mathrm{~cm}^{-2}$ were safe in the hamster pancreas and liver, respectively, and produced lesions of between 3 and $10 \mathrm{~mm}$ in diameter. At these doses, there was no evidence of perforation or obstruction of hollow organs. In a subsequent study in a xenograft model of pancreatic cancer, the extent of verteporfin PDT-induced necrosis was found to be dependent on the aggressiveness of the tumour; at the same light dose, more aggressive AsPC-1 tumours showed a greater amount of necrosis in response to PDT than less aggressive PANC-1 tumours (Samkoe et al, 2010).

These results were sufficient to achieve regulatory approval to conduct a phase I/II study of verteporfin PDT in locally advanced unresectable pancreatic adenocarcinoma (VERTPAC-01).

\section{MATERIALS AND METHODS}

Fifteen patients were treated with verteporfin PDT to confirm its general safety profile, to determine appropriate treatment parameters, and to confirm the safety of PDT if given either before or after conventional palliative chemotherapy or radiotherapy.

The study consisted of two parts. The first part was a single fibre dose escalation study to establish how the extent of necrosis related to the delivered light dose and to choose a suitable light dose for the second, multifibre part of the study.

Patients were eligible if they had a histological or cytological diagnosis of pancreatic adenocarcinoma, proven locally advanced disease confirmed to be unsuitable for surgery at multidisciplinary review, and adequate biliary drainage (serum bilirubin $<2.5 \times$ upper limit of normal). Participants were staged using a pancreatic protocol CT and chest X-ray or CT within 28 days before PDT. It was mandatory for patients to give informed consent, have a negative pregnancy test if appropriate, and an ECOG performance status of $0-2$. Exclusion criteria were porphyria, locally advanced disease involving $>50 \%$ circumference of the duodenum or a major artery (e.g., hepatic and gastroduodenal) within the treatment area, metastatic disease, or ECOG performance status $>2$.

After diagnosis and entry criteria were confirmed, patients received intravenous verteporfin $0.4 \mathrm{mg} \mathrm{kg}^{-1}$ body weight, and prophylactic oral ciprofloxacin $500 \mathrm{mg}$, which was continued for $24 \mathrm{~h}$ after the procedure (three doses in total). Patients were sedated with midazolam and fentanyl and the anterior abdominal wall was infiltrated with local anaesthetic. The tumour was then punctured with a 19-gauge hollow metal needle (Cook Group Inc., Bloomington, IN, USA) under CT guidance by an experienced radiologist. A $0.4-\mathrm{mm}$ core diameter optical fibre with a $1-\mathrm{cm}$ long diffuser tip was inserted through to the tip of the needle and the needle pulled back $10 \mathrm{~mm}$ over the fibre, leaving the diffuser tip in direct contact with the target tumour. An OpadL 690-nm, 0.3-W diode laser was calibrated to deliver $150 \mathrm{~mW} \mathrm{~cm}^{-1}$ along the diffuser tip of the fibre. The tumour was illuminated between 60 and $90 \mathrm{~min}$ after verteporfin administration. The aim of the study was to establish the light dose required to safely produce a zone of necrosis of at least $12 \mathrm{~mm}$ in diameter in a group of three patients treated with each light dose, using a single laser fibre and the chosen dose of verteporfin of $0.4 \mathrm{mg} \mathrm{kg}^{-1}$. The starting light dose was $5 \mathrm{~J}(150 \mathrm{~mW}$ for $33 \mathrm{~s})$. As long as there was no evidence of unacceptable toxicity, the light dose was doubled after each group of three patients until the target size of necrosis was achieved in all three patients in a group, which was at $40 \mathrm{~J}$. At this point, two further patients were treated, one with two fibres (both with $1-\mathrm{cm}$ diffuser tips) and one with three (all with 2-cm diffuser tips). 
Patients remained in subdued lighting for $24 \mathrm{~h}$ after verteporfin injection (or $48 \mathrm{~h}$ in patients with underlying liver disease), followed by re-adaptation to indirect sunlight for increasing periods during the morning and late afternoon of the next day. Bright indoor light was permitted after $24 \mathrm{~h}$ ( $72 \mathrm{~h}$ for underlying liver disease) and exposure to direct sunlight allowed after $48 \mathrm{~h}$ (96 $\mathrm{h}$ for underlying liver disease).

Patients had daily clinical review to assess for potential complications, including abdominal pain, pancreatitis, perforation, biliary obstruction, paralytic ileus, and sepsis. On day 5, a repeat pancreatic protocol CT was performed. The scans were reviewed independently by two consultant radiologists to measure the maximum diameter of PDT-induced necrosis, particularly in the patients treated with a single fibre; and to assess whether the target size of lesion had been achieved. In all patients, the total volume of PDT necrosis was estimated by volume rendering after segmentation on the CT scans.

Treatment-related toxicity was assessed at discharge and at each subsequent follow-up visit. All toxicities were graded according to the National Cancer Institute Common Terminology Criteria for Adverse Events (version 3.0) and were reported to the UCL/UCLH Joint Research Office for documentation. The maximum grade was used for each patient and each toxicity type. The sponsor (University College London) was responsible for monitoring of the trial and provided the regulatory authorities REC (trial reference 06/Q0502/70) and MHRA (trial reference 2006-004097-28) with annual safety reports as well as reporting of any suspected unexpected serious adverse reactions.

An interval CT was performed 4 weeks after PDT, following which patients were able to commence appropriate oncological treatment if they wished. Further treatment with radiotherapy, chemotherapy, and/or retreatment with verteporfin PDT (at the highest safe evaluated dose) was at the discretion of their oncologist and the investigators. Patients continued to be followed for survival data. The primary end point for each patient in the single fibre study was the mean diameter of necrosis achieved around the treatment fibre for a given light dose. Secondary end points were the total volume of PDT necrosis for each treatment, safety, toxicity, tumour response, and overall survival.

\section{RESULTS}

A total of 58 patients were screened for the trial. Fifteen fulfilled the entry criteria and were treated ( 11 male and 4 female; median age 59 (range 47-78)). In one patient, it was concluded that there was too much necrosis present in the tumour before PDT for it to be possible to assess the volume of necrosis induced by the PDT effect, so this patient was excluded from the quantitative analysis, but included in the safety considerations. The median ECOG performance status was 0 (range $0-2$ ).

All patients had proven pancreatic adenocarcinoma on histology or cytology and had a staging CT within 4 weeks of undergoing treatment, which confirmed locally advanced disease, and agreed by a multidisciplinary meeting to be unsuitable for resection surgery. Baseline tumour size was radiologically assessable in 13 patients on the pre-treatment CT and the median diameter in maximal dimension was $40 \mathrm{~mm}$ (range 20-62 mm). Thirteen tumours orginated in the head of the pancreas and two in the body.

All patients were treated according to the protocol; needle placement and light delivery were technically successful in all patients. Representative CT scans of a patient before treatment showing the fibre positioned in the tumour and 5 days later are shown in Figure 1. Thirteen patients were treated in the dose escalation phase of the study and treated with a single laser fibre.
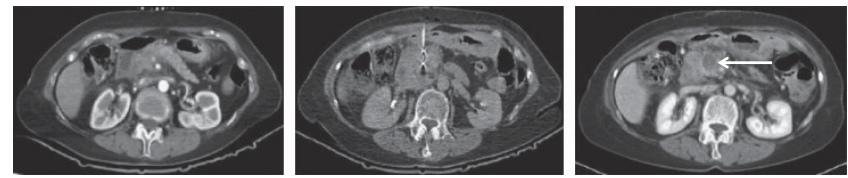

Figure 1. CT scans from patient 13 undergoing verteporfin PDT. The image on the left shows a baseline contrast-enhanced CT with a low attenuation mass in the head of the pancreas. The centre image shows percutaneous needle placement into the tumour. The image on the right shows the day 5 post PDT contrast-enhanced CT with a $2.67-\mathrm{cm}^{3}$ zone of necrosis in the region of the pancreatic head (arrowed).

Of these, three treatments were carried out at each of the 5, 10, 20, and $40 \mathrm{~J} \mathrm{~cm}^{-1}$ settings; a further patient was later also treated at $40 \mathrm{~J} \mathrm{~cm}^{-1}$ as it was an earlier patient in this group in which it was not possible to assess the extent of PDT necrosis due to preexisting necrosis. In the second (multiple fibre) phase of the trial, two patients were treated (one with two and one with three fibres) using $40 \mathrm{~J} \mathrm{~cm}^{-1}$. In the patient treated with three fibres, $20-\mathrm{mm}$ diffuser tip fibres were used. In all other patients $10-\mathrm{mm}$ diffuser tip fibres were used. One patient in the 20-J group was treated on a second occasion 3.5 months after the first PDT with a single fibre delivering $40 \mathrm{~J}$.

No necrosis was seen in the patients treated with $5 \mathrm{~J}$. At $10 \mathrm{~J}$, the diameter of one lesion was over $12 \mathrm{~mm}$, at $20 \mathrm{~J}$, two were over $12 \mathrm{~mm}$ and at $40 \mathrm{~J}$, all three were over $12 \mathrm{~mm}$, so reaching the stopping point defined in the protocol.

Necrosis volumes were determined for all patients (excluding the one with spontaneous necrosis before PDT) using volume rendering after segmentation of the zone of necrosis on the scan slices (Figure 2A-C), and are shown in Figure 2D. The mean volume of necrosis was $0.46 \mathrm{~cm}^{3}$ for the three patients treated at $10 \mathrm{~J}, 1.14 \mathrm{~cm}^{3}$ at $20 \mathrm{~J}$, and $3.48 \mathrm{~cm}^{3}$ at $40 \mathrm{~J}$. In the two patients treated with multiple fibres, the necrosis volumes were 5.3 and $23.2 \mathrm{~cm}^{3}$. The measurements of tumour size before PDT together with the treatment parameters, and volume and mean diameter of necrosis on individual patients are shown in Table 1.

Adverse events. In patients treated with a single fibre, three had mild to moderate abdominal pain shortly after PDT. In two patients this settled on analgesics, although pain persisted in one (the patient who had necrotic tumour before PDT). Another patient had a transient rise in amylase to $234 \mathrm{IUl}^{-1}$ but did not complain of abdominal pain. In all others, the level did not rise above the upper limit of normal $\left(90 \mathrm{IU}^{-1}\right)$. One patient had mild diarrhoea shortly after PDT which settled spontaneously, and another developed persisting steatorrhoea 2 weeks after PDT, neither of which was thought to be directly related to the treatment. No patient had any problems with photosensitivity and there was no evidence of early duodenal obstruction. No patients had overt evidence of bleeding or a fall in haemoglobin.

Both of the patients treated with multiple fibres had evidence on CT of inflammatory change occurring anterior to the pancreas along the needle tract. Both were kept nil by mouth in hospital for observation, although neither had any change in their clinical state and were discharged at 5-10 days after treatment. The inflammatory change was not associated with development of intraabdominal collections or sepsis, and had resolved by the time of the day $28 \mathrm{CT}$.

Follow-up. On RECIST criteria, 11 of the 13 patients in whom the tumour size was assessable pre- and post-treatment had stable disease at 1 month, although 2 of the remainder developed liver metastases at this time. In six patients, stable disease was maintained at 3 months.

Seven adverse events occurred more than 28 days after PDT, which were deemed to be related to tumour progression. 

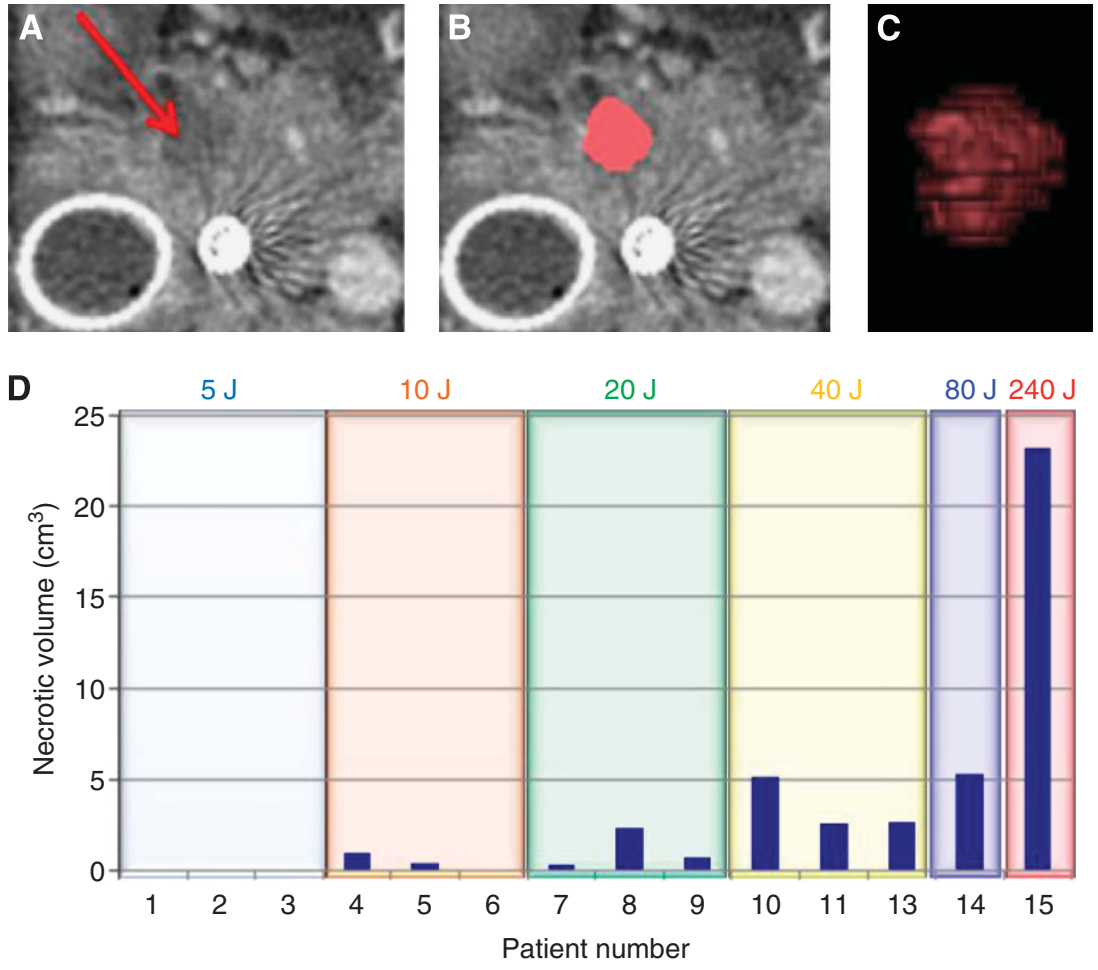

Figure 2. Necrosis measurements following verteporfin PDT in patient 4. (A) A single axial slice of the pancreas from the post-treatment CT scans. The arrow indicates the area of necrosis within the pancreas. (B) The same slice showing segmentation of the necrotic tissue in pink. (C) Volume rendering of segmentation of the necrotic zone. (D) The volume of the necrotic tissue region is shown for each patient in the study, as determined from the segmentation of the post-treatment CT scans. Total energy delivered is shown for each patient. For patient 14, two fibres of $1-\mathrm{cm}$ diffuser length each delivering $40 \mathrm{~J} \mathrm{~cm}^{-1}$ were used. For patient 15, three fibres of $2-\mathrm{cm}$ diffuser length each delivering $40 \mathrm{~J} \mathrm{~cm}{ }^{-1}$ were used. Patient 12 is not included in this figure as necrosis was present before treatment and therefore the effect of treatment could not be assessed.

Five patients developed obstructive jaundice secondary to stent dysfunction and were managed by ERCP and stent change as part of our normal practice. Two patients developed duodenal obstruction, which was managed by endoscopic insertion of selfexpanding metal stents. No other oncological treatments were given within 1 month of PDT. Eight patients received chemotherapy, radiotherapy, or both before PDT and twelve received other oncological treatment following PDT (Table 2). The Kaplan-Meier survival curve is shown in Figure 3. As of September 2013, all but one patient in the study had died (median survival 8.8 months after PDT, range 3.1-25.9 months; median survival from diagnosis 15.5 months, range 4.0-33.6 months).

The one surviving patient (patient 7) was previously enrolled in the PACER trial and treated with conformal radiotherapy and cetuximab 5 months before treatment with PDT at the 20-J energy setting. He was considered to be inoperable on his pre-PDT CT scan as this showed tumour tissue abutting the medial aspect of the superior mesenteric artery (Figure 4A). However, on his scan taken 28 days after PDT, the lesion appeared to have contracted and was no longer directly involving the artery (Figure 4B). As a consequence, he was then deemed to have surgically resectable disease and went on to have a Whipple's pancreaticoduodenectomy. The surgical specimen showed perineural invasion of tumour, but no lymphovascular or lymph-node involvement and the resection margins were clear of tumour (R0 resection). There was dense fibrosis throughout the resected pancreatic tissue with scattered nests of malignant cells. However, there was a central paucicellular area, corresponding to where the light for PDT had been delivered (Figure 5). This zone was smaller than the area of necrosis documented on CT a few days after PDT, consistent with healing as surgery was undertaken 5 weeks after PDT. The patient showed no evidence of recurrence until 36 months after PDT when he was found to have an anterior omental nodule on imaging and was commenced on palliative chemotherapy.

\section{DISCUSSION}

Photodynamic therapy for cancer of the pancreas was first described in 2002 (Bown et al, 2002). That study showed that percutaneous, image guided PDT with the photosensitiser mTHPC (meso-tetrahydroxyphenyl chlorin, temoporfin, Foscan) could produce necrosis in pancreatic cancers with an acceptable level of morbidity. Meso-tetrahydroxyphenyl chlorin is a powerful photosensitiser, but has the considerable disadvantage that it is necessary to wait up to 4 days after giving the drug before delivering the light. It also causes prolonged photosensitivity of skin and eyes to light and patients need to stay out of bright sunlight for up to a month. The aim of this current study was to assess the safety and efficacy of a new generation photosensitiser, using a formal dose escalation approach to establish how the extent of necrosis varied with the delivered light dose. No serious adverse effects were seen using PDT either before or after other oncological treatments, although our protocol dictated an interval of at least a month between treatments.

As expected, the volume of necrosis broadly increased with the amount of energy delivered to the tumour but, as shown in Figure $2 \mathrm{D}$, there was considerable variation in the volume of necrosis within each energy band. These data show that it is difficult to predict the extent of necrosis based just on the dose of photosensitiser, the drug light interval, and the light dose. There may be differences in the pharmacokinetics of the photosensitiser between patients, but it is more likely that there are real-time 


\begin{tabular}{|c|c|c|c|c|c|}
\hline $\begin{array}{l}\text { Patient } \\
\text { number }\end{array}$ & Sex/age & $\begin{array}{c}\text { Baseline } \\
\text { tumour } \\
\text { diam. } \\
(\mathrm{cm})\end{array}$ & $\begin{array}{c}\text { Total } \\
\text { energy } \\
(\mathrm{J})\end{array}$ & $\begin{array}{c}\text { Mean } \\
\text { necrosis } \\
\text { diameter } \\
\text { on day } 5 \\
\text { CT }(\mathrm{cm})\end{array}$ & $\begin{array}{l}\text { Necrosis } \\
\text { volume } \\
\text { on day } 5 \\
\text { CT }\left(\mathrm{cm}^{3}\right)\end{array}$ \\
\hline 1 & $M / 59$ & 4.0 & 5 & 0 & 0 \\
\hline 2 & $M / 76$ & 4.4 & 5 & 0 & 0 \\
\hline 3 & $M / 62$ & $N / A^{a}$ & 5 & 0 & 0 \\
\hline 4 & $\mathrm{~F} / 54$ & 3.8 & 10 & 1.4 & 0.96 \\
\hline 5 & $M / 63$ & 2.8 & 10 & 0.5 & 0.43 \\
\hline 6 & $M / 68$ & 4.2 & 10 & 0 & 0 \\
\hline 7 & $\mathrm{M} / 47$ & 3.8 & 20 & 0.5 & 0.34 \\
\hline 8 & $M / 54$ & 4.0 & 20 & 1.9 & 2.37 \\
\hline 9 & $F / 56$ & 3.5 & 20 & 1.8 & 0.72 \\
\hline 10 & $F / 59$ & $N / A^{a}$ & 40 & 2.2 & 5.17 \\
\hline 11 & $\mathrm{M} / 57$ & 3.8 & 40 & 2.1 & 2.61 \\
\hline 12 & $\mathrm{M} / 78$ & 6.5 & 40 & N/A & $N / A^{b}$ \\
\hline 13 & $F / 69$ & 2.0 & 40 & 2.1 & 2.67 \\
\hline 14 & $M / 56$ & 3.7 & 80 & 3.1 & 5.3 \\
\hline 15 & $M / 65$ & 5.3 & 240 & 4.1 & 23.2 \\
\hline \multicolumn{6}{|c|}{ 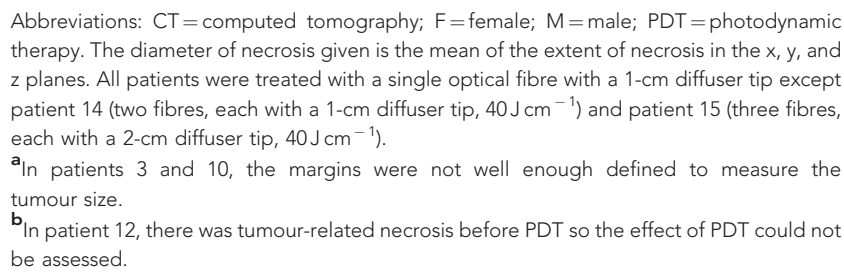 } \\
\hline
\end{tabular}

Table 2. Oncological treatment given before and after PDT

\begin{tabular}{|l|c|c|c|}
\hline & All & Female & Male \\
\hline No prior treatment & 7 & 1 & 6 \\
\hline Prior chemotherapy & 3 & 0 & 3 \\
\hline Prior chemo-radiotherapy & 3 & 1 & $2^{\mathbf{a}}$ \\
\hline Prior immunotherapy & $2^{\mathbf{b}}$ & 2 & 0 \\
\hline No treatment after PDT & 3 & 1 & 2 \\
\hline Chemotherapy after PDT & 8 & 2 & 6 \\
\hline Chemo-radiotherapy after PDT & 1 & 0 & 1 \\
\hline Immunotherapy after PDT & $2^{\mathbf{b}}$ & 1 & 1 \\
\hline $\begin{array}{l}\text { Whipple's pancreaticoduodenectomy + chemotherapy } \\
\text { after PDT }\end{array}$ & 1 & 0 & 1 \\
\hline $\begin{array}{l}\text { Abbreviation: PDT = photodynamic therapy. } \\
\text { a One patient was previously enrolled in the PACER Trial (Radical radiotherapy with } \\
\text { concurrent Cetuximab monoclonal antibody therapy). } \\
\text { b Four patients were enrolled in the Telovac Trial (GV1001 vaccine vs gemcitabine + } \\
\text { capecitabine in a phase III trial for advanced and metastatic pancreatic cancer). No } \\
\text { oncological treatment was given less than a month before or after PDT . }\end{array}$ \\
\hline
\end{tabular}

variations related to the tissue and vascular perfusion around a fibre positioned interstitially that influence how deeply the light penetrates.

In both of the patients treated with multiple fibres, the first follow-up CT scan at day 5 showed unexpected inflammation anterior to the pancreas. Investigation of this ex vivo demonstrated

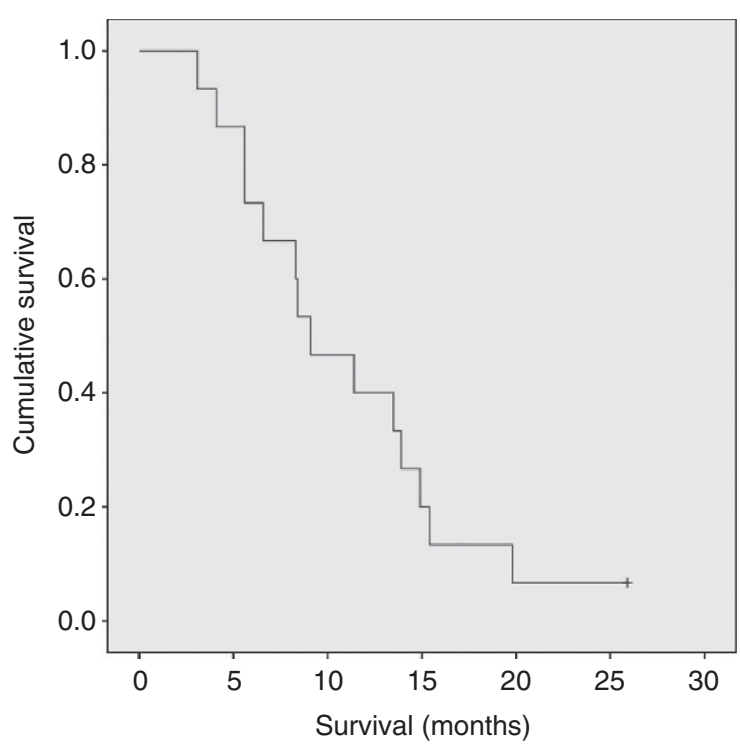

$\begin{array}{llllllll}\text { Number at risk } & 15 & 13 & 7 & 3 & 1 & 1 & 1\end{array}$

Figure 3. Kaplan-Meier curve showing the cumulative survival from treatment with PDT in all patients.
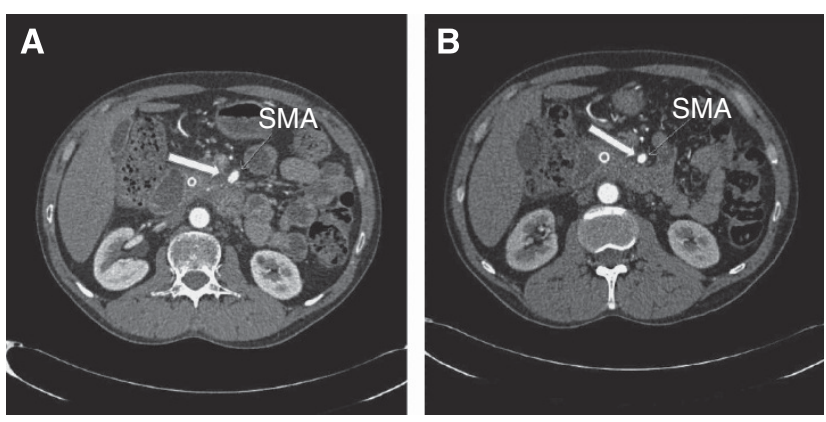

Figure 4. CT scan from patient 7 who had a successful Whipple's resection following PDT. (A) Image from CT before PDT showing tumour abutting the superior mesenteric artery (block arrow). (B) Four weeks after PDT, the repeat CT scan at the same level suggested that there was a clear tissue plane between the artery and the tumour (block arrow), so rendering him suitable for surgery. As these scans were at the level of maximum tumour involvement of the artery, they are not exactly at the same level as the PDT necrosis, which is not visible on scan (B).

that the likely explanation lay in a problem with the fibres. When the fibres were examined in the physics laboratory, it was apparent that the emission of light from the fibres was not limited to the diffuser tip but extended for up to about $1 \mathrm{~cm}$ proximal to the tip. The light intensity in this region was considerably less than in the intended emission zone, however, with multiple fibres the overlapping fields resulted in this area receiving a higher light dose than in patients treated with a single fibre and the tissue in this region would not be expected to be immune from a PDT effect. In the mTHPC paper (Bown et al, 2002), an area of normal pancreas was treated inadvertently in one patient when a fibre slipped after positioning. A clear area of necrosis was seen in the normal pancreas, but this healed safely without sequelae, as demonstrated on CT after 1 month. These observations further support previous reports of how PDT is not highly tumour selective, but that treated areas in a range of normal tissues can heal safely without a significant loss of structure or function (Anand et al, 2012; Bown, 2013). 


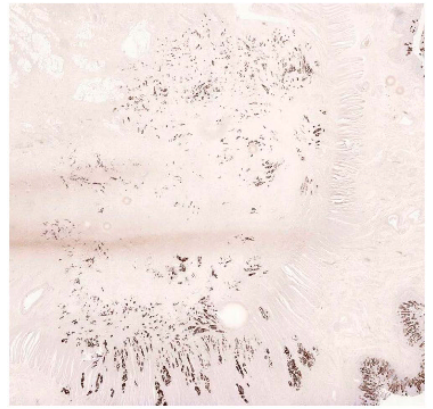

Figure 5. Pancytokeratin immunohistochemistry section of pancreatic cancer from patient 7 who underwent a Whipple's pancreaticoduodenectomy 5 weeks after PDT. The patient received radiotherapy 5 months before PDT, causing fibrosis over a wide part of the gland in addition to that inherent to pancreatic adenocarcinomas. However, there is a clearly identifiable central area in this section of the tumour with a markedly reduced number of cells, compared with the surrounding tumour. This corresponds to the PDT-treated area.

The median survival from treatment with PDT in this study was similar to a comparable group of patients treated conventionally, with a median survival from diagnosis of 15.5 months. However, this was a highly selected non-randomised group who had both prior and subsequent oncological treatments so conclusions on survival advantage cannot be made. Nevertheless, it is of interest that after PDT, one patient was re-staged and underwent a successful R0 resection. This may have been related to volume shrinkage due to resorption of necrotic tissue in the treated area of cancer, as was documented in three cases in our earlier pancreatic PDT study (Bown et al, 2002). In this regard, there is increasing interest in the potential use of loco-regional treatments as part of a multimodality approach to the management of pancreatic cancer. Indeed, the recently presented SCALOP trial showed that induction chemotherapy followed by chemoradiotherapy could be given safely and effectively (Mukherjee et al, 2013). Radiofrequency ablation has also been used in phase I settings, both via a surgical and endoscopic approach but randomised trials are lacking (Pezzilli et al, 2011; Arcidiacono et al, 2012). Irreversible electroporation is an emerging non-thermal ablative technique that uses electrodes, placed in the tumour, to deliver up to $3 \mathrm{kV}$ of direct current to induce the formation of nanoscale pores within the cell membrane of the targeted tissue, causing apoptosis (Martin et al, 2012; Narayanan et al, 2012). The US Food and Drug Administration have recently approved the technique for use in the pancreas.

In conclusion, we have shown in this phase I/II study that verteporfin PDT-induced tumour necrosis in locally advanced pancreatic cancer is feasible and safe. The low adverse event profile does suggest that, alongside systemic treatments, there is a role for further studies of PDT for the local treatment of pancreatic adenocarcinoma or possibly for other focal pancreatic neoplasms such as neuroendocrine tumours and pancreatic cystic neoplasms.

\section{ACKNOWLEDGEMENTS}

We would like to thank the patients, and their families, without whom this trial would not have been possible. We also thank the UCL Joint Research Office for facilitating the conduct of the study, and the independent data monitoring committee (Professor Hugh Barr (Chair), Mr Hemant Kocher, and Dr Gary Middleton). This work was supported by NIH grant P01 CA084203, Killing Cancer and the UCLH Charitable Foundation. It was also partly supported by the UCLH/UCL Comprehensive Biomedical Centre which receives a proportion of funding from the Department of Health's National Institute for Health Research (NIHR) Biomedical Research Centres funding scheme.

\section{CONFLICT OF INTEREST}

The authors declare no conflict of interest.

\section{REFERENCES}

Anand S, Ortel BJ, Pereira SP, Hasan T, Maytin EV (2012) Biomodulatory approaches to photodynamic therapy for solid tumors. Cancer Lett 326(1): $8-16$.

Arcidiacono PG, Carrara S, Reni M, Petrone MC, Cappio S, Balzano G, Boemo C, Cereda S, Nicoletti R, Enderle MD, Neugebauer A, von Renteln D, Eickhoff A, Testoni PA (2012) Feasibility and safety of EUS-guided cryothermal ablation in patients with locally advanced pancreatic cancer. Gastrointest Endosc 76(6): 1142-1151.

Ayaru L, Wittmann J, Macrobert AJ, Novelli M, Bown SG, Pereira SP (2007) Photodynamic therapy using verteporfin photosensitization in the pancreas and surrounding tissues in the Syrian golden hamster. Pancreatology 7(1): 20-27.

Barr H, Tralau CJ, Boulos PB, MacRobert AJ, Tilly R, Bown SG (1987) The contrasting mechanisms of colonic collagen damage between photodynamic therapy and thermal injury. Photochem Photobiol 46(5): $795-800$.

Bown SG (2013) Photodynamic therapy for photochemists. Philos Trans A Math Phys Eng Sci 371(1995): 20120371.

Bown SG, Rogowska AZ, Whitelaw DE, Lees WR, Lovat LB, Ripley P, Jones L, Wyld P, Gillams A, Hatfield AW (2002) Photodynamic therapy for cancer of the pancreas. Gut 50(4): 549-557.

Chen B, Pogue BW, Goodwin IA, O'Hara JA, Wilmot CM, Hutchins JE, Hoopes PJ, Hasan T (2003) Blood flow dynamics after photodynamic therapy with verteporfin in the RIF-1 tumor. Radiat Res 160(4): 452-459.

Chen B, Pogue BW, Hoopes PJ, Hasan T (2005a) Combining vascular and cellular targeting regimens enhances the efficacy of photodynamic therapy. Int J Radiat Oncol Biol Phys 61(4): 1216-1226.

Chen B, Pogue BW, Zhou X, O'Hara JA, Solban N, Demidenko E, Hoopes PJ, Hasan T (2005b) Effect of tumor host microenvironment on photodynamic therapy in a rat prostate tumor model. Clin Cancer Res 11(2 Pt 1): 720-727.

Conroy T, Desseigne F, Ychou M, Bouche O, Guimbaud R, Becouarn Y, Adenis A, Raoul JL, Gourgou-Bourgade S, de la Fouchardiere C, Bennouna J, Bachet JB, Khemissa-Akouz F, Pere-Verge D, Delbaldo C, Assenat E, Chauffert B, Michel P, Montoto-Grillot C, Ducreux M (2011)

FOLFIRINOX versus gemcitabine for metastatic pancreatic cancer. $N$ Engl $J$ Med 364(19): 1817-1825.

Cronin-Fenton DP, Erichsen R, Mortensen FV, Dikinis S, Norgaard M, Jacobsen J (2011) Pancreatic cancer survival in central and northern Denmark from 1998 through 2009: a population-based cohort study. Clin Epidemiol 3(Suppl 1): 19-25.

Cunningham D, Chau I, Stocken DD, Valle JW, Smith D, Steward W, Harper PG, Dunn J, Tudur-Smith C, West J, Falk S, Crellin A, Adab F, Thompson J, Leonard P, Ostrowski J, Eatock M, Scheithauer W, Herrmann R, Neoptolemos JP (2009) Phase III randomized comparison of gemcitabine versus gemcitabine plus capecitabine in patients with advanced pancreatic cancer. J Clin Oncol 27(33): 5513-5518.

Eeson G, Chang N, McGahan CE, Khurshed F, Buczkowski AK, Scudamore CH, Warnock GL, Chung SW (2012) Determination of factors predictive of outcome for patients undergoing a pancreaticoduodenectomy of pancreatic head ductal adenocarcinomas. HPB 14(5): 310-316.

Fingar VH, Kik PK, Haydon PS, Cerrito PB, Tseng M, Abang E, Wieman TJ (1999) Analysis of acute vascular damage after photodynamic therapy using benzoporphyrin derivative (BPD). Br J Cancer 79(11-12): 1702-1708.

Glidden MD, Celli JP, Massodi I, Rizvi I, Pogue BW, Hasan T (2012) Image-based quantification of benzoporphyrin derivative uptake, localization, and photobleaching in 3D tumor models, for optimization of PDT parameters. Theranostics 2(9): 827-839. 
Gong Z, Holly EA, Bracci PM (2011) Survival in population-based pancreatic cancer patients: San Francisco Bay area, 1995-1999. Am J Epidemiol 174(12): 1373-1381.

He J, Edil BH, Cameron JL, Schulick RD, Hruban RH, Herman JM, Zheng L, Iacobuzio-Donahue C, Ahuja N, Pawlik TM, Wolfgang CL (2013) Young patients undergoing resection of pancreatic cancer fare better than their older counterparts. J Gastrointest Surg 17(2): 339-344.

Houle JM, Strong A (2002a) Clinical pharmacokinetics of verteporfin. J Clin Pharmacol 42(5): 547-557.

Houle JM, Strong HA (2002b) Duration of skin photosensitivity and incidence of photosensitivity reactions after administration of verteporfin. Retina 22(6): 691-697.

Kindler HL, Niedzwiecki D, Hollis D, Sutherland S, Schrag D, Hurwitz H, Innocenti F, Mulcahy MF, O'Reilly E, Wozniak TF, Picus J, Bhargava P, Mayer RJ, Schilsky RL, Goldberg RM (2010) Gemcitabine plus bevacizumab compared with gemcitabine plus placebo in patients with advanced pancreatic cancer: phase III trial of the Cancer and Leukemia Group B (CALGB 80303). J Clin Oncol 28(22): 3617-3622.

Kinsella TJ, Seo Y, Willis J, Stellato TA, Siegel CT, Harpp D, Willson JK, Gibbons J, Sanabria JR, Hardacre JM, Schulak JP (2008) The impact of resection margin status and postoperative CA19-9 levels on survival and patterns of recurrence after postoperative high-dose radiotherapy with 5-FU-based concurrent chemotherapy for resectable pancreatic cancer. Am J Clin Oncol 31(5): 446-453.

Kuroda T, Kumagi T, Yokota T, Seike H, Nishiyama M, Imai Y, Inada N, Shibata N, Imamine S, Okada S, Koizumi M, Yamanishi H, Azemoto N, Miyaike J, Tanaka Y, Tatsukawa H, Utsunomiya H, Ohno Y, Miyake T, Hirooka M, Furukawa S, Abe M, Ikeda Y, Matsuura B, Hiasa Y, Onji M (2013) Improvement of long-term outcomes in pancreatic cancer and its associated factors within the gemcitabine era: a collaborative retrospective multicenter clinical review of 1,082 patients. BMC Gastroenterol 13: 134 .

Lin GC, Tsoukas ML, Lee MS, Gonzalez S, Vibhagool C, Anderson RR, Kollias N (1998) Skin necrosis due to photodynamic action of benzoporphyrin depends on circulating rather than tissue drug levels: implications for control of photodynamic therapy. Photochem Photobiol 68(4): 575-583.

Lo VC, Akens MK, Moore S, Yee AJ, Wilson BC, Whyne CM (2012) Beyond radiation therapy: photodynamic therapy maintains structural integrity of irradiated healthy and metastatically involved vertebrae in a pre-clinical in vivo model. Breast Cancer Res Treat 135(2): 391-401.

Luo J, Xiao L, Wu C, Zheng Y, Zhao N (2013) The incidence and survival rate of population-based pancreatic cancer patients: shanghai cancer registry 2004-2009. PLoS ONE 8(10): e76052.

Martin 2nd RC, McFarland K, Ellis S, Velanovich V (2012) Irreversible electroporation therapy in the management of locally advanced pancreatic adenocarcinoma. J Am Coll Surg 215(3): 361-369.

Moore MJ, Goldstein D, Hamm J, Figer A, Hecht JR, Gallinger S, Au HJ, Murawa P, Walde D, Wolff RA, Campos D, Lim R, Ding K, Clark G, Voskoglou-Nomikos T, Ptasynski M, Parulekar W (2007) Erlotinib plus gemcitabine compared with gemcitabine alone in patients with advanced pancreatic cancer: a phase III trial of the National Cancer Institute of Canada Clinical Trials Group. J Clin Oncol 25(15): 1960-1966.

Mukherjee S, Hurt CN, Bridgewater J, Falk S, Cummins S, Wasan H, Crosby T, Jephcott C, Roy R, Radhakrishna G, McDonald A, Ray R, Joseph G, Staffurth J, Abrams RA, Griffiths G, Maughan T (2013) Gemcitabine-based or capecitabine-based chemoradiotherapy for locally advanced pancreatic cancer (SCALOP): a multicentre, randomised, phase 2 trial. Lancet Oncol 14(4): 317-326.

Narayanan G, Hosein PJ, Arora G, Barbery KJ, Froud T, Livingstone AS, Franceschi D, Rocha Lima CM, Yrizarry J (2012) Percutaneous irreversible electroporation for downstaging and control of unresectable pancreatic adenocarcinoma. J Vasc Interv Radiol 23(12): 1613-1621.

Panjehpour M, DeNovo RC, Petersen MG, Overholt BF, Bower R, Rubinchik V, Kelly B (2002) Photodynamic therapy using Verteporfin (benzoporphyrin derivative monoacid ring A, BPD-MA) and $630 \mathrm{~nm}$ laser light in canine esophagus. Lasers Surg Med 30(1): 26-30.

Pezzilli R, Serra C, Ricci C, Casadei R, Monari F, D’Ambra M, Minni F (2011) Radiofrequency ablation for advanced ductal pancreatic carcinoma: is this approach beneficial for our patients? A systematic review. Pancreas 40(1): 163-165.

Samkoe KS, Chen A, Rizvi I, O’Hara JA, Hoopes PJ, Pereira SP, Hasan T, Pogue BW (2010) Imaging tumor variation in response to photodynamic therapy in pancreatic cancer xenograft models. Int J Radiat Oncol Biol Phys 76(1): 251-259.

Sant M, Allemani C, Santaquilani M, Knijn A, Marchesi F, Capocaccia R (2009) EUROCARE-4. Survival of cancer patients diagnosed in 1995-1999. Results and commentary. Eur J Cancer 45(6): 931-991.

Turrini O, Paye F, Bachellier P, Sauvanet A, Sa Cunha A, Le Treut YP, Adham M, Mabrut JY, Chiche L, Delpero JR (2013) Pancreatectomy for adenocarcinoma in elderly patients: postoperative outcomes and long term results: a study of the French Surgical Association. Eur J Surg Oncol 39(2): 171-178.

Von Hoff DD, Ervin T, Arena FP, Chiorean EG, Infante J, Moore M, Seay T, Tjulandin SA, Ma WW, Saleh MN, Harris M, Reni M, Dowden S, Laheru D, Bahary N, Ramanathan RK, Tabernero J, Hidalgo M, Goldstein D, Van Cutsem E, Wei X, Iglesias J, Renschler MF (2013) Increased survival in pancreatic cancer with nab-paclitaxel plus gemcitabine. $N$ Engl J Med 369(18): 1691-1703.

von Wichert G, Seufferlein T, Adler G (2008) Palliative treatment of pancreatic cancer. J Dig Dis 9(1): 1-7.

Wu L, Murphy RP (1999) Photodynamic therapy: a new approach to the treatment of choroidal neovascularization secondary to age-related macular degeneration. Curr Opin Ophthalmol 10(3): 217-220.

This work is published under the standard license to publish agreement. After 12 months the work will become freely available and the license terms will switch to a Creative Commons AttributionNonCommercial-Share Alike 3.0 Unported License. 